\title{
Ion-beam induced current in high-resistance materials
}

\author{
V.I. Yukalov ${ }^{1,2}$ and E.P. Yukalova ${ }^{1,3}$ \\ ${ }^{1}$ Instituto de Fisica de São Carlos, Universidade de São Paulo \\ Caixa Postal 369, São Carlos, São Paulo 13560-970, Brazil \\ 2 Bogolubov Laboratory of Theoretical Physics \\ Joint Institute for Nuclear Research, Dubna 141980, Russia \\ 3 Department of Computational Physics \\ Laboratory of Computing Techniques and Automation \\ Joint Institute for Nuclear Research, Dubna 141980, Russia
}

\begin{abstract}
The peculiarities of electric current in high-resistance materials, such as semiconductors or semimetals, irradiated by ion beams are considered. It is shown that after ion-beam irradiation an unusual electric current may arise directed against the applied voltage. Such a negative current is a transient effect appearing at the initial stage of the process. The possibility of using this effect for studying the characteristics of irradiated materials is discussed. A new method for defining the mean projected range of ions is suggested.
\end{abstract}

41.75.-i, 72.20.-i

\section{INTRODUCTION}

The irradiation of semiconductors and insulators by ion beams is a tool that is frequently used for studying or modifying the electric properties of these high-resistance materials (see e.g. [1,2] and references therein). As s result of ion irradiation, heavily doped charged layers can be formed in a sample [3], with the density of ions in the layer reaching $10^{20} \mathrm{~cm}^{-3}$. The formation of strongly nonuniform charge distributions is the standard situation in the ionbeam irradiation, while for achieving a uniform volume concentration of implanted ions special tricks are required [4].

Under the influence of ion irradiation, electric properties of high-resistance materials can be essentially changed $[1,2,5]$, with additional peculiarities resulting from a nonuniform distribution of charge carriers [6-9]. High-resistance materials mean, as usual, the materials with a very poor concentration of carriers, such as semiconductors, semimetals, or insulators that, being irradiated, acquire conducting properties $[1,2,5]$. The electron transport in such materials can be well described in the semiclassical drift-diffusion approach $[10,11]$. The existence of the implantation induced damages can be effectively taken into account by means of characteristic parameters entering the drift-diffusion equations $[10,11]$, for instance, by specifying the resistivity or mobility $[1,2]$.

When an energetic ion strikes a solid surface, there is a probability of electron capture resulting in the neutralization of a part of implanted ions that become electrically inactive. However, the neutralized ions can easily be activated again by irradiating the material with laser beams [12-15].

In the present paper, we study the electric transport in high-resistance materials with a strongly nonuniform initial distribution of charge carriers, which can be formed by ion-beam irradiation. As follows from the above discussion, it is always possible to prepare such conditions, e.g. employing laser activation [12-15], that the injected ions could be the principal charge carriers. For concreteness, we consider below positive ions, though this assumption is not principal and negative ions could be treated in the same footing.

\section{PECULIARITIES OF ELECTRIC TRANSPORT}

The transport properties of high-resistance materials, such as extrinsic semiconductors, are usually described by the drift-diffusion approach $[10,11]$ consisting of the continuity and Poisson equations, respectively,

$$
\frac{\partial \rho}{\partial t}+\vec{\nabla} \cdot \vec{j}+\frac{\rho}{\tau}=0, \quad \varepsilon \vec{\nabla} \cdot \vec{E}=4 \pi \rho,
$$


where $\rho=\rho(\vec{r}, t)$ is the charge density of carriers; $\vec{j}=j(\vec{r}, t)$ is the electric current density; $\tau$ is the relaxation time; and $\varepsilon$ is the dielectric permittivity. The total current density is

$$
\vec{j}_{t o t}=\vec{j}+\frac{\varepsilon}{4 \pi} \frac{\partial \vec{E}}{\partial t}, \quad \vec{j}=\mu \rho \vec{E}-D \vec{\nabla} \rho,
$$

where $\mu$ is the mobility of carriers and $D=(\mu / \varepsilon) k_{B} T$ is the diffusion coefficient. The average current through the considered sample is given by the integral

$$
\vec{J}(t)=\frac{1}{V} \int \vec{j}_{t o t}(\vec{r}, t) d \vec{r}
$$

over the sample volume $V$.

Let us consider a plane sample of the thickness $L$ and area $A$, which is biased with an external constant voltage $V_{0}>0$. Because of the plane symmetry, the three-dimensional picture will be reduced everywhere below to the one-dimensional description. For what follows, it is convenient to simplify the notation passing to dimensionless quantities. The return to dimensional quantities can be easily done as follows. We keep in mind that the coordinate $x$ is measured in units of the thickness $L$ and the time $t$, in units of the transit time $\tau_{0}$, so that for returning to the corresponding dimensional variables one has to make the substitution

$$
x \rightarrow \frac{x}{L}, \quad t \rightarrow \frac{t}{\tau_{0}}, \quad \tau_{0} \equiv \frac{L^{2}}{\mu V_{0}} .
$$

For other physical quantities the return to dimensional units is done by accomplishing the following transitions: for the diffusion coefficient,

$$
D \rightarrow \frac{D}{D_{0}}, \quad D_{0} \equiv \mu V_{0},
$$

for the electric field,

$$
E \rightarrow \frac{E}{E_{0}}, \quad E_{0} \equiv \frac{V_{0}}{L},
$$

for the total accumulated charge,

$$
Q \rightarrow \frac{Q}{Q_{0}}, \quad Q_{0} \equiv \varepsilon A E_{0}=\frac{\varepsilon A V_{0}}{L}
$$

for the charge density,

$$
\rho \rightarrow \frac{\rho}{\rho_{0}}, \quad \rho_{0} \equiv \frac{Q_{0}}{A L}=\frac{\varepsilon V_{0}}{L^{2}},
$$

and for the electric current,

$$
j \rightarrow \frac{j}{j_{0}}, \quad j_{0} \equiv \frac{Q_{0}}{A \tau_{0}}=\frac{\varepsilon V_{0}}{L \tau_{0}},
$$

with the same transformation for the average current (3), $J \rightarrow J / j_{0}$.

Employing the dimensionless notation, for the plane case considered, we have from equations (1)

$$
\frac{\partial \rho}{\partial t}+\frac{\partial}{\partial x}(\rho E)-D \frac{\partial^{2} \rho}{\partial x^{2}}+\frac{\rho}{\tau}=0, \quad \frac{\partial E}{\partial x}=4 \pi \rho,
$$

where the space and time variables are such that

$$
0<x<1, \quad t>0 .
$$

The total current density (2) reads

$$
j_{t o t}=\rho E-D \frac{\partial \rho}{\partial x}+\frac{1}{4 \pi} \frac{\partial E}{\partial t} .
$$


The condition that the sample is biased with a constant external voltage now writes

$$
\int_{0}^{1} E(x, t) d x=1
$$

Note that the parameters $\varepsilon$ and $\mu$ do not appear in Eqs. (4) because of the usage of the dimensionless units.

An initial condition to the continuity equation is defined by the distribution of ions after the irradiation process. The distribution of implanted species can be modelled by the Gaussian form

$$
\rho(x, 0)=\frac{Q}{Z} \exp \left\{-\frac{(x-a)^{2}}{2 b}\right\},
$$

in which $0<a<1$ and

$$
Q=\int_{0}^{1} \rho(x, 0) d x, \quad Z=\int_{0}^{1} \exp \left\{-\frac{(x-a)^{2}}{2 b}\right\} d x .
$$

The distribution centre, $a$, is close to the mean projected range of ions, although may be not exactly coinciding with it.

Our aim is to study the behaviour of the electric current through the sample,

$$
J(t)=\int_{0}^{1} j_{t o t}(x, t) d x
$$

as a function of time, when the initial distribution of carriers is given by the form (7). In particular, we shall show that the nonuniformity of the initial distribution may lead to quite unexpected behaviour of the current (8) when it turns against the applied voltage becoming negative, This will be shown to be the result of the solution of the transport equations (4).

First of all, let us emphasize that the occurrence of the negative current, if any, can be due only to a nonuniform distribution of carriers. Really, the current (8), with the current density (5), can be written as

$$
J(t)=\int_{0}^{1} \rho(x, t) E(x, t) d x+D[\rho(0, t)-\rho(1, t)] .
$$

If $\rho(x, t)$ does not depend on $x$, then Eq. (9), with the help of the voltage condition (6), immediately shows that the current is positive since we are dealing with a positive charge density $\rho$. As far as the diffusion and relaxation terms in the continuity equation tend to smooth the initial nonuniform distribution of carriers, this tells us that the negative current can happen only at the initial stage of the process while the charge density $\rho(x, t)$ is yet essentially nonuniform. The initial stage means that $t \ll 1$, when the influence of the diffusion and relaxation terms is yet negligible. In other words, the favouring conditions for the occurrence of the negative electric current are $D \ll 1$ and $\tau \gg 1$. It is worth recalling here that, according to the discussion given in the Introduction, the influence of the implantation-induced damages is assumed to be taken into account by the corresponding values of parameters in the transport equations and that the implanted ions are assumed to be prepared as electrically active, which can be done by means of laser irradiation [12-15].

To demonstrate explicitly that the negative current really can occur, let us analyse the case of a very narrow layer of injected ions, such that the standard deviation $b$, also called the straggling [1], is small, $b \ll 1$. Then distribution (7) is close to

$$
\rho(x, 0)=Q \delta(x-a)
$$

In the beginning of the process, when $t \approx 0$, we have from Eqs. $(9)$ and $(10)$

$$
J(0)=Q E(a, 0)
$$

Integrating the second equation in Eq. (4) yields

$$
E(a, 0)=1+4 \pi Q\left(a-\frac{1}{2}\right) .
$$

With these conditions, the current (8) becomes negative provided that 


$$
4 \pi Q\left(\frac{1}{2}-a\right)>1
$$

From here, the inequality

$$
a<\frac{1}{2}-\frac{1}{4 \pi Q}
$$

follows for the location of the ion layer. Since this location is inside the sample, we also have the inequality

$$
Q>\frac{1}{2 \pi} \quad(0<a<1)
$$

for the initial charge.

The above analysis demonstrates that the negative current really can occur provided some special conditions, as (12) and (13), hold true. To our knowledge, there have been no experimental measurements demonstrating the appearance of such a negative current. Therefore the picture we describe here is a theoretical prediction of a novel effect. The occurrence of this negative current is very sensitive to the characteristics of the irradiated sample as well as to the initial nonuniform distribution of carriers, which suggests the possibility of using this effect for studying the mentioned properties. For instance, the mean projected range of irradiating ions could be measured in this way. This can be realized as follows. Assume that for an ion-irradiated material we observed the occurrence of the negative current $J(0)$ at initial time. Let us compare the observed value $J(0)$ with formula (9) where we have to substitute the distribution (7). The peak of the latter is close to the mean projected range of the irradiating ions. With the given $\rho(x, 0)$, the right-hand side of expression (9) can be explicitly calculated. This is because the electric field satisfying Eq. (4), with the voltage condition (6), can be presented as the functional

$$
E(x, t)=1+4 \pi\left[Q(x, t)-\int_{0}^{1} Q(x, t) d x\right]
$$

of the density $\rho(x, t)$ entering through

$$
Q(x, t)=\int_{0}^{x} \rho\left(x^{\prime}, t\right) d x^{\prime}
$$

Hence, for a given $\rho(x, 0)$, the electric field $E(x, 0)$ is uniquely defined by the above functional. Thus, equating the calculated $J(0)$ from formula (9) with the corresponding measured value, we obtain an equation for the dimensionless mean projected range $a$. For example, in the case of a narrow ion layer, we find

$$
a=\frac{1}{2}-\frac{1}{4 \pi Q}+\frac{J(0)}{4 \pi Q^{2}} .
$$

Returning to dimensional units, for the mean projected range $\lambda=a L$ we obtain

$$
\lambda=\frac{L}{2}-\frac{\varepsilon A V_{0}}{4 \pi Q}+\frac{\varepsilon A^{2} L^{2}}{4 \pi \mu Q^{2}} J(0) .
$$

This formula directly relates the mean projected range of ions, $\lambda$, with the known parameters of the system and the measured initial current $J(0)$. In deriving Eq. (14), we have only used the fact that the initial distribution of ions is narrow, $b \ll 1$, while the current $J(0)$ can, in general, be of any sign. The advantage of using the effect of the negative electric current is that the latter always requires a relatively narrow initial distribution. Therefore, as soon as we observe a negative current $J(0)$, we can employ formula (14) as a realistic approximation for the ion mean projected range.

When the initial ion layer is not narrow and the current $J(0)$ is not necessary negative, so that the dependence of the formulas on the standard deviation $b$ becomes important, then two situations can happen. One is when $b$ is known from other experiments. Then, in order to find the mean projected range, one should proceed exactly as is described above equating the measured and calculated currents $J(0)$. If the straggling $b$ is not known, it can be found in the following way. Assume that at the initial time $t=0$ there appears the negative current. Since this is a transient effect, existing only in a finite time interval $0 \leq t \leq t_{0}$, there is some time $t=t_{0}$ when the current changes its sign becoming normal, i.e. positive, for $t>t_{0}$. At the same time $t=t_{0}$, the current then is zero, $J\left(t_{0}\right)=0$. Therefore, the latter equation, with the given experimentally measured $t_{0}$, may serve as an additional condition defining the straggling $b$. 
It may also happen that the injected ion layer is narrow but located close to the surface, so that $a \sim b$. Then formula (14) can be corrected by taking into account the second term in Eq. (9), which gives for the mean projected range

$$
\lambda=\frac{L}{2}-\frac{\varepsilon A}{4 \pi Q}\left[V_{0}+\frac{A L}{\mu Q}(D \Delta \rho-L J)\right]
$$

where

$$
J \equiv J(0), \quad \Delta \rho \equiv \rho(0,0)-\rho(1,0)
$$

To find the electric current (8) for arbitrary times, we need to solve equations (4), with the voltage condition (6) and the initial condition (7). Those should also be complimented by boundary conditions which can be taken in the Neumann form [10]. We have accomplished such numerical calculations whose results are presented in the figures, where all values are given in dimensionless units, and the conditions $D \ll 1$ and $\tau \gg 1$, favouring the occurrence of the negative current are assumed. The figures correspond to the setup explained in detail in the Introduction and specified in the present section.

Fig. 1 shows the dependence of the electric current $J(t)$ on time for the varying location $a$ of the initial distribution peak of ions. Formulas (14) and, respectively, (15) for the mean projected range are valid with a very good accuracy, with an error being less than $1 \%$. In order to distinguish the lines, they are numbered.

Fig. 2 presents the electric current as a function of time for different widths of the initial distribution of ions. Formulas (14) and (15) are again very accurate for the negative current $J(0)$. But when the current $J(0)$ is positive and at the same time, the initial distribution is not narrow, then Eqs. (14) and (15) are not valid, as it should be.

Fig. 3 demonstrates the time dependence of the electric current for different values of the charge $Q$. Since $b \ll 1$, formulas (14) and (15) work perfectly for both cases, for the negative current, as in Fig. 3, as well as for positive current, as in Fig. 4; the errors being not more than around 1\%. The current in Fig. 4 becomes positive because condition (13) does not hold. Increasing the accumulated charge $Q$, so that condition (13) becomes true, the negative current appears provided that condition (12) is satisfied, as is clearly illustrated in Fig. 5.

\section{DISCUSSION}

We have shown that in high resistance materials irradiated by ion beams an unusual transient effect of the negative electric current can occur. This effect can be used for studying the characteristics of irradiated materials as well as those of irradiating ions. For instance, the mean projected range of ions can be measured whose value is well approximated by formula (15). This new way of measuring the ion mean projected range is, certainly, not the sole possible, but it can provide additional information being complimented by other experimental methods. Throughout the paper, we have been talking about ions. But, as is clear, the same consideration concerns any kind of charged particles, say electrons, positrons, or muons.

It is worth stressing that here we have advanced a theoretical proposal for observing and employing a novel effect. The general prerequisites for realizing the latter look, as follows from the above discussions, achievable. Moreover, there exist so many types of various high-resistance materials, such as semiconductors, semimetals, and insulators, there are so many ways of fabricating such materials with specially designed characteristics, and also there are so many methods of varying the properties of these materials by involving additional external sources, as electromagnetic fields or laser beams, that the feasibility of preparing the desired conditions for realizing the suggested effect looks quite realistic. Even if this realization is difficult today, it might be accomplished in future. The history shows that practically any effect that is possible in principle sooner or later becomes realizable in experiment.

Although our main aim in this paper has been to advance a theoretical proposal for the principal possibility of observing a novel effect, we have also paid attention to the feasibility of realizing it experimentally. In addition to the above discussions, we would like to touch several other important points that could substantiate the feasibility of observing the considered effect.

First, the space charge due to the accumulated ions will act against further implantation, and the ion doses will certainly be limited. At the same time, to observe the effect of negative electric current, one needs to reach some threshold value for the accumulated charge, as in the inequality (13). In order to understand how the latter condition can be satisfied, it is necessary to rewrite it in the corresponding dimensional units introduced in the beginning of Sec. 2 , in this case, in the units of $Q_{0}$. This gives us the condition

$$
2 \pi \varepsilon A \frac{V_{0}}{L} Q>1 .
$$


From here, it is evident that this condition can always be satisfied for any given charge $Q$, which can be achieved by increasing either the sample area $A$ or the applied voltage $V_{0}$. Of course, for a given sample, one has to increase the voltage $V_{0}$.

The implanted ions do not form a homogeneous layer and their distribution may be not exactly Gaussian. In this paper we consider the Gaussian distribution (7) which usually describes well the profile of implanted ions. However this form is not compulsory for realizing the negative-current effect. The latter persists as well for other distributions, for which one needs only that the straggling $b$ be less than the projected range $a$.

For the realization of the effect, it is also not necessary that the ion distribution be centered at the mean projected range of ions. For simplicity, we called the distribution center $a$ the projected range. However, if these two do not coincide, all consideration remains valid with merely slightly changing the terminology, so that $a$ is to be called the distribution center.

To measure currents through the sample, one has to take into account that near contacts there often occurs the space charge build-up caused by electron or hole injection from contacts. The current due to the carriers injected from contacts can mask the current produced by ions. How to ascertain that the considered effect is caused by the implanted ions? This problem can be easily resolved by measuring the current through the sample before ion irradiation. Then one may study the influence of contacts on creating electric currents. In this way, one can always separate the influence of contacts from physical effects resulting from ion irradiation.

In conclusion, we do not see principal difficulties for realizing the negative-current effect. And, as follows from the above discussions, different technical problems seem to be resolvable. Being realized, this effect will make it possible to have an additional tool for studying the transport properties of high-resistance materials as well as to measure characteristics related to irradiated ions.

\section{Acknowledgement}

We appreciate financial support from the São Paulo State Research Foundation.

[1] S. Prawer, R. Kalish, M. Adel, and V. Richter, J. Appl. Phys. 61, 4492 (1987).

[2] S. Coffa, F. Priolo, and A. Battaglia, Phys. Rev. Lett. 70, 3756 (1993).

[3] R.M. Bayazitov et al., Nucl. Instrum. Methods Phys. Res. B 122, 35 (1997).

[4] V.F. Reutov, Nucl. Instrum. Methods Phys. Res. B 140, 433 (1998).

[5] V.I. Yukalov, Ionizing Radiation Effects on Properties of Dielectrics and Semiconductors, edited by M.I. Ryazanov (Atomizdat, Moscow, 1979), p. 217.

[6] A.I. Rudenko and V.I. Yukalov, Investigation of Surface and Volume Properties of Solids by Particle Interactions, edited by M.I. Ryazanov (Energoizdat, Moscow, 1981), p. 78.

[7] V.I. Yukalov, JINR Rapid Commun. 7, 51 (1985).

[8] V.I. Yukalov and E.P. Yukalova, Phys. Lett. A 236, 113 (1997).

[9] V.I. Yukalov, E.P. Yukalova, and M.R. Singh, Phys. Rev. B 59, 10111 (1999).

[10] C.M. Snowden, Introduction to Semiconductor Device Modelling (World Scientific, Singapore, 1986).

[11] K. Seeger, Semiconductor Physics (Springer, Berlin, 1989).

[12] S. Prawer, R. Kalish, and M. Adel, Appl. Phys. Lett. 48, 1585 (1986).

[13] D. Bäuerle, Laser Processing and Chemistry (Springer, Berlin, 1996).

[14] V.I. Yukalov and E.P. Yukalova, Laser Phys. 7, 1076 (1997).

[15] H. Opower, Phys. Stat. Sol. A 166, 555 (1998). 


\section{Figure Captions}

\section{Fig. 1.}

Electric current through the sample as a function of time for the parameters $Q=1, b=0.05$, and different initial locations of the ion layer: $a=0.1$ (curve 1, solid line), $a=0.25$ (curve 2, long-dashed line), $a=0.5$ (curve 3 , short-dashed line), and $a=0.75$ (curve 4 , dotted line).

\section{Fig. 2 .}

The dependence of the electric current on time for $Q=0.5, a=0.1$, and varying widths of the initial ion distribution: $b=0.05$ (curve 1 , solid line), $b=0.1$ (curve 2 , long-dashed line), and $b=0.5$ (curve 3 , short-dashed line).

\section{Fig. 3.}

Electric current as a function of time for $a=0.25, b=0.05$ and different charges: $Q=3$ (curve 1 , solid line), $Q=1$ (curve 2, long-dashed line), and $Q=0.5$ (curve 3, short-dashed line).

\section{Fig. 4.}

The time dependence of the electric current for $Q=0.1, b=0.05$, and two different initial locations of the ion distribution: $a=0.1$ (curve 1 , solid line) and $a=0.25$ (curve 2, long-dashed line).

Fig. 5 .

Electric current vs. time for $Q=0.5, b=0.1$, and varying initial locations of the peak of the ion distribution: $a=0.1$ (curve 1 , solid line), $a=0.25$ (curve 2 , long-dashed line), $a=0.5$ (curve 3 , short-dashed line), and $a=0.75$ (curve 4, dotted line). 